Manuelle Medizin 2016 $54: 366$

DOI 10.1007/s00337-016-0174-z

(C) Springer-Verlag

Berlin Heidelberg 2016

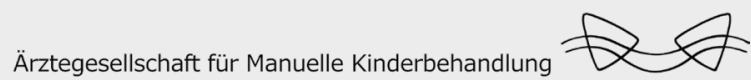

\& Atlastherapie e.V.

\section{Ärztegesellschaft für Manuelle Kinderbehandlung} und Atlastherapie (ÄMKA) e.V.

in Kooperation mit dem Dr. Karl-Sell-Ärzteseminar Neutrauchburg (MWE) e.V.

$\begin{array}{ll}\text { Anna-von-Borries-Str. } 1 & \text { V.i.S.d.P.: } \\ \text { D-730625 Hannover } & \text { Ruth Kamping } \\ \text { Fax:0511-5354675 } & \text { Georgstr. } 46 \\ \text { info@aegamk.de } & \text { 30159 Hannover }\end{array}$

\title{
Kursprogramm 2016
}

\section{Kursprogramm Hannover 2016}

Kursort: Annastift Hannover

Kursleiter: Dr. A. Thren, R. Kamping, Dr. P. Weng,

Dr. M. Ruprecht

Kinderkurs A: $\quad$ Diagnostikkurs $1 \quad$ 16.09.-17.09.2016

Kinderkurs B: $\quad$ Diagnostikkurs 2 18.11.-19.11.2016

Atlastherapie nach Arlen Kurs 1: $\quad$ 14.09.-15.09.2016

Atlastherapie nach Arlen Kurs 2: $\quad$ 16.11.-17.11.2016

\section{Anmeldung ausschließlich über:}

Diakonische Dienste Hannover GmbH

Akademie für Fort- und Weiterbildung

Anna-von-Borries-Str. 1-7, 30625 Hannover

Info-Tel: 0511-5354662, Fax: 0511-5354672

info@ddh.akademie.de,www.ddh-akademie.de

\section{Kursprogramm Isny-Neutrauchburg 2016}

Kursleiter: Dr. M. Riedel, Dr. M. Habring, Dr. A. Sammer, Dr. P. Mantl

$\begin{array}{lll}\text { Kurs C } & \text { Therapiekurs 1 } & \text { 15.09.-17.09.2016 } \\ \text { Kurs D } & \text { Therapiekurs 2 } & \text { 24.11.-26.11.2016 }\end{array}$

\section{Anmeldung:}

MWE-Sekretariat

Riedstr. 5, 88316 Isny-Neutrauchburg

Tel: 07562-9718-0, Fax: 07562-9718-22

info@manuelle-mwe.de,www.manuelle-mwe.de

\section{Atlaskurs 1}

Besondere Bedeutung des zervikookzipitalen Überganges (Kopfgelenke 1), praktische Übungen.

\section{Atlaskurs 2}

Kopfgelenk (Teil 2), klinische und physiologische Aspekte, therapeutischer Ansatz, Simulator-Training. Schriftl. Test.

\section{Kinder-Kurs A Diagnostikkurs 1}

Neurophysiologische und manualmedizinische Untersuchung im Säuglingsalter: frühkindliche Entwicklung, Reflexologie, sensomotorische Integration, funktionelle und segmentale Befundung der sensorischen Schlüsselregionen.

\section{Kinder-Kurs B Diagnostikkurs 2}

Sensomotorische Dysfunktion im Kindesalter, ADS, Therapiegrundsätze bei idiopathischer Skoliose. Infantile Zerebralparese: Propädeutik und Untersuchungstechnik.

\section{Kinder-Kurs C Therapiekurs 1}

Manuelle Impuls - und Weichteiltechniken bei Säuglingen und Kindern an Wirbelsäule und Extremitäten.

\section{Kinder-Kurs D Therapiekurs 2}

Manuelle Impuls- und Weichteiltechniken an Wirbelsäule und Extremitäten (Teil 2), Atlastherapie bei Säuglingen und Kindern.

Examen. Zertifikat

Die Atlaskurse können auch unabhängig von den Kinderkursen besucht werden. Ein kombinierter Besuch ist jedoch sinnvoll, da die Atlaskurse Voraussetzung und wichtiger Bestandteil der Ausbildung in manueller Kinderbehandlung sind. So sind die Atlaskurse den Kinderkursen $\mathrm{A}+\mathrm{B}$ vorangestellt.

Voraussetzung für die Kursteilnahme ist die abgeschlossene Weiterbildung in Manueller Medizin. Es werden nur approbierte Ärzte zugelassen. Die Diagnostikkurse sind Voraussetzung für die Teilnahme an den Therapiekursen.

Basisseminar "General Movements" nach der Prechtl-Methode Eine diagnostische Methode zur funktionellen Beurteilung der Integrität des Nervensystems. Referentin: Prof. Dr. Christa Einspieler, 03.11.-06.11.2016, Annastift Hannover Anmeldung: DDH Hannover (s. Kursort Hannover) 